\title{
APPROXIMATE SOLUTIONS TO FIRST AND SECOND ORDER QUASILINEAR EVOLUTION EQUATIONS VIA NONLINEAR VISCOSITY
}

\author{
JUAN R. ESTEBAN AND PIERANGELO MARCATI
}

\begin{abstract}
We shall consider a model problem for the fully nonlinear parabolic equation

$$
u_{t}+F\left(x, t, u, D u, \varepsilon D^{2} u\right)=0
$$

and we study both the approximating degenerate second order problem and the related first order equation, obtained by the limit as $\varepsilon \rightarrow 0$. The strong convergence of the gradients is provided by semiconcavity unilateral bounds and by the supremum bounds of the gradients. In this way we find solutions in the class of viscosity solutions of Crandall and Lions.
\end{abstract}

\section{INTRODUCTION}

The theory of viscosity solutions to fully nonlinear equations has been widely developed in the recent past, in connection with the study of several first and second order nonlinear P.D.E.'s arising in many different branches of pure and applied mathematics. Let us recall, for instance the deterministic and stochastic control theory, the theory of Hamilton-Jacobi equations, the geometrical optics analysis and more recently the motion of level sets by mean curvature and its applications to the theory of image processing, cf. [ALM, ES1, ES2, CGG, GGIS].

Within this framework very general results concerning the existence and the uniqueness of the solutions can be obtained (see for instance the book [L] and the survey $[\mathrm{CIL}]$ ) and we intend to investigate the various intimate connections between the methods of approximating nonsmooth solutions via very regular solutions, in particular how to pass into the limit into highly nonlinear terms.

The analysis involves different degrees of difficulty, in particular the loss of regularity of the limit (e.g., the kinks formation for the Hamilton-Jacobi equations) and the possible degeneracy of the higher order terms (e.g., the porous medium equation). So we are going to investigate here a model problem which will bring together all those levels of difficulties.

Received by the editors December 9, 1991.

1991 Mathematics Subject Classification. Primary 35K55; Secondary 35K65.

First author partially supported by E.E.C. Contract SC1-0019-C.

CNR-Visiting Professor at the Department of Pure and Applied Mathematics, University of L'Aquila.

Second author partially supported by CNR-GNAFA and MURST. 
The previous approximation problems can be set in some general form as follows (we refer to [CIL] for the standard definitions of the theory of viscosity solutions). Let us consider the fully nonlinear parabolic equation

$$
u_{t}+F\left(x, t, u, D u, \varepsilon D^{2} u\right)=0,
$$

where $\varepsilon$ is a positive parameter and $F$ is a continuous function (including the case of degenerate elliptic $F)$. The unknown $u$ is $u=u(x, t)$ for $x \in \mathbf{R}^{N}$ and $t>0, D u=\left(D_{1} u, \ldots, D_{N} u\right)$ represents the spatial gradient of $u$, and $D^{2} u$ corresponds to the matrix of second order derivatives of $u$ with respect to $x$.

Two important questions can be addressed, the former concerns the approximation of the eventually degenerate problem (1.1) by nondegenerate equations, the latter the limit as $\varepsilon \rightarrow 0$ in (1.1), to approximate the case of the completely degenerate $F=F(x, t, u, D u, 0)$. In both of these two issues, it is important to obtain relevant informations about the first and second order derivatives of the solution.

A partial answer to these questions could be obtained by using the general approach, via the uniqueness and the stability properties of viscosity solutions: sup-inf convolutions and Perron's method (see for instance [CIL, BP]). Since in general this approach does not provide estimates and convergence of the derivatives, we are motivated to use a more traditional P.D.E. approach.

This paper wishes to be a first step in the general program outlined above. We consider a specific nonlinear model problem which includes some basic features though not all, of the general case.

Let $H(X)$ be a convex function of $X \in \mathbf{R}^{N}$. We will study how to approximate the following initial value problems: the first is the quasilinear degenerate parabolic problem

$$
\begin{aligned}
& u_{t}+H(D u)=\varepsilon \operatorname{div}\left(|D u|^{p-1} D u\right) \quad \text { in } \mathbf{R}^{N} \times(0, T), \\
& u(x, 0)=u_{0}(x) \quad \text { in } \mathbf{R}^{N},
\end{aligned}
$$

where $p>1$. The second is regarding the limit as $\varepsilon \rightarrow 0$ in the previous problem, namely the quasilinear first order problem

$$
\begin{cases}u_{t}+H(D u)=0 & \text { in } \mathbf{R}^{N} \times(0, T), \\ u(x, 0)=u_{0}(x) & \text { in } \mathbf{R}^{N}\end{cases}
$$

The problem $\left(\mathrm{P}_{\varepsilon}\right)$ can be considered as a "vanishing viscosity" approximation to problem $(\mathrm{P})$. For instance, if the growth of $H(X)$ is controlled by $|X|^{p+1}$ for some $p>1$, we balance this behaviour by introducing in $\left(P_{\varepsilon}\right)$ a nonlinear viscosity second order operator, namely the $p$-Laplacian operator

$$
\Delta_{p}(u)=\operatorname{div}\left(|D u|^{p-1} D u\right),
$$

whose principal part is of the order of $|D u|^{p-1}$.

The approximation of $(P)$ by using the model problem $\left(\mathrm{P}_{\varepsilon}\right)$ has interest by itself which goes beyond the general program described previously. Indeed, the construction of finite difference schemes to solve $(P)$ takes some advantages by using a nonlinear "artificial viscosity" (see for instance the book [RM]), which was proposed by von Neumann many years ago. Moreover, into some extent, the results in this paper generalize those in [M] for the case $N=1$, where 
methods of compensated compactness are used and some numerical schemes are proposed.

Another important fact about the model problem $\left(\mathrm{P}_{\varepsilon}\right)$ concerns the growth rate of the first order term (i.e., the exponent $p+1$ larger than 2 in the hamiltonian $H$ ) and the growth rate of order $p-1$ in the diffusion coefficient of $\Delta_{p}(u)=\operatorname{div}\left(|D u|^{p-1} D u\right)$. Actually two units of difference between both rates of growth is the usual balance between the first and second terms, which appears in the regularity theory for quasilinear parabolic P.D.E.'s. We recall that this balance is required to establish gradient bounds for the solutions. When this condition is violated, some counterexamples can be found in [LSU]. So the operator $\Delta_{p}(u)=\operatorname{div}\left(|D u|^{p-1} D u\right)$ seems to be a natural choice to generalize the vanishing viscosity method for hamiltonians which grow like $|D u|^{p+1}$. It is also worth mentioning that in such a case similarity solutions of the form $u\left(|x| / t^{1 /(p+1)}\right)$ can be computed, and the ratio between $x$ and $t$ is the same both for $\left(\mathrm{P}_{\varepsilon}\right)$ and $(\mathrm{P})$.

In the case of $H(X)=|X|^{p+1}$, problem $\left(\mathrm{P}_{\varepsilon}\right)$ is in strong connection with the theory of nonnegative solutions to doubly nonlinear equations of the form $u_{t}=\Delta_{p}\left(u^{m}\right)$ as $m p=1$, that are investigated in [EV3] and in the case $N=1$ in [EV1].

In order to deal with the degeneracy in the gradient dependence of the problem $\left(\mathrm{P}_{\varepsilon}\right)$ we introduce, on the line of [EV2], a second approximation procedure which leads us to study a problem of the form

$$
\left(\mathbf{P}_{\varepsilon, \delta}\right) \quad\left\{\begin{array}{l}
u_{t}+H_{\delta}(D u)=\varepsilon \operatorname{div}\left(\phi_{\delta}(|D u|) D u\right) \text { in } \mathbf{R}^{N} \times(0, T), \\
u(x, 0)=u_{0}(x) \text { in } \mathbf{R}^{N},
\end{array}\right.
$$

where $\delta>0$, and the hamiltonian $H_{\delta}$ is a very simple approximation of $H$. We construct explicitly the nonlinear function $\phi_{\delta}=\phi_{\delta}(r)$ for $r \geq 0$, such that $\phi_{\delta}(r)=r^{p-1}$ for $r \geq \delta$ and $\phi_{\delta}(r)=\phi_{\delta}(0)$ for $r \leq \delta / 2$. One then has an operator

which satisfies

$$
\Delta_{p}^{\delta}(u)=\operatorname{div}\left(\phi_{\delta}(|D u|) D u\right)
$$

$$
\Delta_{p}^{\delta}(u)=\Delta_{p}(u) \text { for }|D u| \geq \delta,
$$

as well as

$$
\Delta_{p}^{\delta}(u)=\phi_{\delta}(0) \Delta(u) \text { for }|D u| \leq \delta / 2
$$

The problem $\left(\mathrm{P}_{\varepsilon, \delta}\right)$ is no longer degenerate and the regularity theory of quasilinear parabolic equations provides smooth solutions of $\left(\mathrm{P}_{\varepsilon, \delta}\right)$, cf. [LSU].

In $\S 2$ we establish the bound

$$
\Delta_{p}^{\delta}\left(u_{\varepsilon, \delta}\right)=\operatorname{div}\left(\phi_{\delta}\left(\left|D u_{\varepsilon, \delta}\right|\right) D u_{\varepsilon, \delta}\right) \leq k / t, \quad \text { in } \mathbf{R}^{N} \times(0,+\infty),
$$

where $k=p N / \lambda$ and $\lambda>0$ is related to the convexity properties of $H(X)$. So one has a semiconcavity estimate which is independent both of $\varepsilon$ and $\delta$, and moreover it follows

$$
\Delta_{p}(u)=\operatorname{div}\left(|D u|^{p-1} D u\right) \leq k / t,
$$

for the solutions of problem $\left(\mathbf{P}_{\varepsilon}\right)$ and of problem $(\mathbf{P})$. Semiconcavity inequalities involving the $p$-Laplacian operator have been first established in [EV2] and our proof is based on some techniques of that paper. We wish to stress the hyperbolic nature of this estimate, which comes from the convexity of the 
hamiltonian $H$ and is, for this reason, obviously not true as $H=0$. This fact is strongly related to the Oleinik's entropy conditions for scalar conservation laws with convex flux, when $N=1$. Several estimates of this nature have been obtained for the Laplace operator (namely the case $p=1$ in (1.2)) in the framework of the Hamilton-Jacobi equations, for hamiltonians having a quadratic growth. The semiconcavity bounds for the eikonal equation found an interesting application in the theory of the porous media equation, by considering the gradient square term in the "pressure" equation (see for instance [A]). Some other semiconcavity estimates have been obtained in [CLS], for a more general class of hamiltonians.

Two types of gradient bounds are obtained in $\S 3$. The former is a universal bound for the gradient which depends explicitly on the semiconcavity estimate and the oscillation amplitude of the solution. It is established by the classical Bernstein method [B, LSU, S], although the application given here cannot be obtained as a consequence of these papers. Because of the term $|D u|^{p-1}$ in the principal part of the $p$-Laplacian operator (1.2), the coefficients of the linearized problem depend not only on $u$ and $D u$ but also on the second order derivatives of $u$. The previously obtained semiconcavity bound is a crucial argument in providing a control of these terms. This kind of difficulty does not appear when dealing either with the usual vanishing viscosity or with other situations which are linear in the second order term of the equation.

The latter is a more traditional gradient bound obtained from the behaviour as $|x| \rightarrow \infty$ of the gradient at $t=0$, via a maximum principle technique. However, it is not essential in the study of the convergence that will be done later.

Section 4 deals with the construction of some upper and lower barriers which allow to control the oscillation amplitude of the solution. Although we do not make this computation explicitly, they can be used to attack this problem with Perron's method, as outlined in [CIL]. The barriers' construction has been done by a suitable modification of the explicit formulas for the Hamilton-Jacobi equations [L, BE].

We finally deal with the limits, first as $\delta \rightarrow 0$ then as $\varepsilon \rightarrow 0$, in the framework of viscosity solutions. We use the local bound on $\left|D u_{\varepsilon, \delta}\right|$ and the semiconcavity $\Delta_{p}^{\delta}\left(u_{\varepsilon, \delta}\right) \leq k / t$ to show equiboundedness of $\Delta_{p}^{\delta}\left(u_{\varepsilon, \delta}\right)$ in the space of measures. By Minty's method, we can pass to the limit inside $\Delta_{p}^{\delta}$. The two estimates above also give some strong compactness of $\Delta_{p}^{\delta}\left(u_{\varepsilon, \delta}(\cdot, t)\right)$ in $W_{\text {loc }}^{-1, s}\left(\mathbf{R}^{N}\right), 1<s<$ $+\infty$. The monotonicity and the coercivity of $\Delta_{p}^{\delta}$ provide the strong convergence of the gradient. The limit solution is still a "viscosity solution" because of wellknown stability properties. The definition of viscosity solutions is well known, we refer to [CIL, $\S 8$; CEL, IL] for details.

The uniqueness of the solutions to $\left(\mathrm{P}_{\varepsilon}\right)$ and $(\mathrm{P})$ in the class of viscosity solutions is provided by the theory developed by Ishii-Lions [IL], Crandall [C], or by Theorem 8.2 in [CIL].

\section{Semiconcavity}

In this section we prove the semiconcavity estimate for the solution $u=$ $u_{\varepsilon}(x, t)$ of the initial value problem $\left(\mathrm{P}_{\varepsilon}\right)$. Let $H(X)$ to be a smooth and 
convex function of $X \in \mathbf{R}^{N}$, such that its second order derivatives

$$
H^{i j}(X)=\partial^{2} H(X) / \partial X_{i} \partial X_{j}
$$

satisfy

$$
\lambda|X|^{p-1}|Y|^{2} \leq H^{i j}(X) Y_{i} Y_{j} \leq \Lambda|X|^{p-1}|Y|^{2},
$$

for some $p>1$, some $0<\lambda \leq \Lambda$ and every $X, Y \in \mathbf{R}^{N}$. We have

Theorem 1. Let $H(X)$ satisfy (2.1). Then the unique viscosity solution $u=$ $u_{\varepsilon}(x, t)$ of $\left(\mathbf{P}_{\varepsilon}\right)$ satisfies $\Delta_{p}(u) \leq k / t$ as a measure in $\mathbf{R}^{N} \times(0, \infty)$. The constant $k$ is $k=p N / \lambda$.

1. To present the basic arguments in the proof of Theorem 2, we first proceed formally.

a. To begin with, we develop the term

$$
\Delta_{p}(u)=D_{i}\left[|D u|^{p-1} D_{i} u\right]
$$

at the points $(x, t)$ where $D u(x, t) \neq 0$. This leads to the expression

$$
\Delta_{p}(u)=|D u|^{p-1} E_{i j} D_{i j} u,
$$

where we have defined

$$
E_{i j}=E_{i j}(D u)=\left(\delta_{i j}-d_{i} d_{j}\right)+p d_{i} d_{j}
$$

and

$$
d_{i}=D_{i} u /|D u|, \quad \text { for } i, j=1,2, \ldots, N .
$$

By using the coefficients $E_{i j}$ we can also write the identity

$$
D_{j}\left[|D u|^{p-1} D_{i} u\right]=|D u|^{p-1} E_{i k} D_{j k} u,
$$

for $i=1,2, \ldots, N$ and $j \in\{1,2, \ldots, N ; t\}$.

b. Let $u$ satisfy

$$
u_{t}+H(D u)=\varepsilon \Delta_{p}(u) \text { in } \mathbf{R}^{N} \times(0, \infty),
$$

and consider the linear operator

$$
\mathscr{L}(\varphi)=\varphi_{t}-\varepsilon D_{i}\left[|D u|^{p-1} E_{i j}(D u) D_{j} \varphi\right]+H^{k}(D u) D_{k} \varphi .
$$

(Here and in the sequel, we use the notation $H^{k}(X)=\partial H(X) / \partial X_{k}$.)

We will show that $\theta=\Delta_{p}(u)$ satisfies

$$
\mathscr{L}(\theta) \leq-\theta^{2} / k
$$

Then, Theorem 2 will follow from the Maximum Principle: since for $\bar{\theta}=k / t$ we have $\mathscr{L}(\bar{\theta})=-\bar{\theta}^{2} / k$ and $\bar{\theta}(x, 0) \equiv+\infty \geq \theta(x, 0)$, then

$$
\theta=\Delta_{p} u \leq \bar{\theta}=k / t .
$$

c. Let us now compute $\mathscr{L}(\theta)$. Thanks to (2.4), we have

$$
\theta_{t}=D_{i}\left[|D u|^{p-1} E_{i j} D_{j} u_{t}\right]
$$

Equation (2.5) for $u$ gives

$$
D_{j} u_{t}=\varepsilon D_{j} \theta-H^{k} D_{j k} u,
$$


which together with $(2.8)$ yields

$$
\begin{aligned}
\theta_{t} & =\varepsilon D_{i}\left[|D u|^{p-1} E_{i j} D_{j} \theta\right]-D_{i}\left[H^{k}|D u|^{p-1} E_{i j} D_{j k} u\right] \\
& =\varepsilon D_{i}\left[|D u|^{p-1} E_{i j} D_{j} \theta\right]-D_{i}\left[H^{k} D_{k}\left[|D u|^{p-1} D_{i} u\right]\right]
\end{aligned}
$$

Therefore, we obtain

$$
\begin{aligned}
\mathscr{L}(\theta) & =H^{k} D_{k}\left[D_{i}\left[|D u|^{p-1} D_{i} u\right]\right]-D_{i}\left[H^{k} D_{k}\left[|D u|^{p-1} D_{i} u\right]\right] \\
& =-D_{i}\left[H^{k}\right] D_{k}\left[|D u|^{p-1} D_{i} u\right] \\
& =-|D u|^{p-1} \operatorname{Trace}\left[\left(\mathscr{H} D^{2} u\right)\left(\mathscr{E} D^{2} u\right)\right]
\end{aligned}
$$

where we have defined the matrices $\mathscr{H}=\left(H^{i j}\right), \mathscr{E}=\left(E_{i j}\right)$, and $D^{2} u=\left(D_{i j} u\right)$.

d. Now we show (2.7) in the simple case $H(X)=|X|^{p+1}$. We have

$$
\theta=\Delta_{p}(u)=|D u|^{p-1} \operatorname{Trace}\left(\mathscr{E} D^{2} u\right)
$$

and

$$
\mathscr{L}(\theta)=-(p+1)|D u|^{2(p-1)} \operatorname{Trace}\left[\left(\mathscr{E} D^{2} u\right)^{2}\right] .
$$

Therefore we obtain

$$
\mathscr{L}(\theta) \leq-\frac{p+1}{N}|D u|^{2(p-1)}\left[\operatorname{Trace}\left(\mathscr{E} D^{2} u\right)\right]^{2}=-\frac{p+1}{N} \theta^{2} .
$$

e. The proof of (2.7) for general $H(X)$ satisfying (2.1) is achieved by looking at the canonical form of the matrix $\mathscr{E}$.

Let us consider the quantities

$$
\begin{array}{ll}
Q=\sum_{i, j=1}^{N}\left(D_{i j} u\right)^{2}, & B^{2}=\sum_{i, j=1}^{N}\left(d_{i} D_{i j} u\right)^{2}, \\
T=\Delta u=\sum_{i=1}^{N} D_{i i} u, & A=\sum_{i, j=1}^{N} d_{i} d_{j} D_{i j} u .
\end{array}
$$

We have from (2.2) and (2.3) that

$$
\theta=\Delta_{p}(u)=|D u|^{p-1}[(T-A)+p A],
$$

and from Cauchy-Schwarz inequality

$$
\frac{1}{N} \theta^{2} \leq|D u|^{2(p-1)}\left[\frac{1}{N-1}(T-A)^{2}+p^{2} A^{2}\right] .
$$

As in Lemma 1 of [EV2], we also have

Lemma 2.1. (a) An orthogonal transformation $\mathscr{C}$ can be made such that

$$
\mathscr{E}=\mathscr{C} \operatorname{diag}\{p, 1, \ldots, 1\} \mathscr{C}^{T} .
$$

Moreover, $\vec{d}=\left(d_{1}, \ldots, d_{N}\right)$ is an eigenvector of $\mathscr{E}$ corresponding to the eigenvalue $p$.

(b) Let $\theta=\Delta_{p}(u)$. Then

$$
\theta^{2} / N \leq|D u|^{2(p-1)}\left[\left(Q+A^{2}-2 B^{2}\right)+p^{2} A^{2}\right] .
$$


To prove (2.7), we define $\overline{\mathscr{H}}=\left(\bar{H}^{i j}\right)=\mathscr{C}^{T} \mathscr{H} \mathscr{C}, \mathscr{U}=\left(U_{i j}\right)=\mathscr{C}^{T} D^{2} u \mathscr{C}$, and apply Lemma 2.1 (a) to get that $(2.9)$ reads

$$
\mathscr{L}(\theta)=-|D u|^{p-1} \bar{H}^{j k}\left\{p U_{1 j} U_{1 k}+\sum_{i=2}^{N} U_{i j} U_{i k}\right\} .
$$

At this point, we use the left inequality in our hypotheses $(2.1)$ on $H$. It implies

$$
\begin{aligned}
\mathscr{L}(\theta) & \leq-\lambda|D u|^{2(p-1)}\left\{p \sum_{j=1}^{N}\left(U_{1 j}\right)^{2}+\sum_{i=2}^{N} \sum_{j=1}^{N}\left(U_{i j}\right)^{2}\right\} \\
& \leq-\lambda|D u|^{2(p-1)}\left\{p B^{2}+\left[Q+A^{2}-2 B^{2}\right]+\left[B^{2}-A^{2}\right]\right\} .
\end{aligned}
$$

Finally, thanks to $A^{2} \leq B^{2}$ and Lemma $2.1(\mathrm{~b})$ we obtain

$$
\mathscr{L}(\theta) \leq-\frac{\lambda}{p}|D u|^{2(p-1)}\left\{\left[Q+A^{2}-2 B^{2}\right]+p^{2} A^{2}\right\} \leq-\frac{\lambda}{p N} \theta^{2} .
$$

2a. We remark that solutions of (2.5) do not possess the properties of regularity and boundedness required to perform directly the computations above and to use the Maximum Principle for (2.6). Actually, equation (2.5) fails to be uniformly parabolic at the points $(x, t)$ where $D u(x, t)=0$. As a consequence,

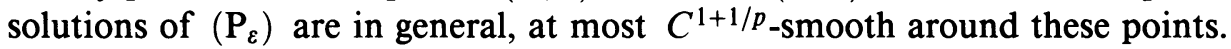

Thus, to prove Theorem 1 we are led to regularize suitably the $p$-Laplacian operator $\Delta_{p}(u)=\operatorname{div}\left[|D u|^{p-1} D u\right]$, and consider instead the problem

$$
\begin{aligned}
& u_{t}+H_{\delta}(D u)=\varepsilon \operatorname{div}\left[\phi_{\delta}(|D u|) D u\right] \text { in } \mathbf{R}^{N} \times(0, \infty), \\
& u(x, 0)=u_{0}(x) \text { in } \mathbf{R}^{N} .
\end{aligned}
$$

Here $\delta>0$ and we construct a smooth $\phi_{\delta}=\phi_{\delta}(r)$ such that $\phi_{\delta}(r)=r^{p-1}$ for $r \geq \delta$ and $\phi_{\delta}(r)=\phi_{\delta}(0)>0$ for $r \approx 0$. The precise definition and properties of $\phi_{\delta}$ are presented next.

Lemma 2.2. There exists a nondecreasing and $C^{\infty}[0, \infty)$ function $\phi_{\delta}=\phi_{\delta}(r)$, satisfying

(i) $\phi_{\delta}(r)=r^{p-1}$ for $r \geq \delta$,

(ii) $\phi_{\delta}(r)=\phi_{\delta}(0)>0$ for $0 \leq r \leq \delta / 2$,

(iii) $r^{1-p} \phi_{\delta}(r)$ is nondecreasing for $0<r \leq \delta$.

Proof of Lemma 2.2. Consider a monotone and $C^{\infty}[0, \infty)$ function $\sigma_{\delta}(r)$ such that $\sigma_{\delta}(r)=1$ for $0 \leq r \leq \delta / 2$ and $\sigma_{\delta}(r)=p$ for $r \geq \delta$. We want $\sigma_{\delta}(r)$ to represent "the exponent" of $r \phi_{\delta}(r)$. To this aim, we define $\phi_{\delta}(r)$ by

$$
1+r \phi_{\delta}^{\prime}(r) / \phi_{\delta}(r)=\sigma_{\delta}(r) \text {. }
$$

This gives

$$
\phi_{\delta}(r)=\phi_{\delta}(0) \exp \left\{\int_{\delta / 2}^{r} \frac{\sigma_{\delta}(s)-1}{s} d s\right\},
$$

and the value of $\phi_{\delta}(0)$ can be chosen to obtain (i). The other assertions of the lemma are then easy to check.

Remark 2.3. With this definition of $\phi_{\delta}$, the operator

$$
\Delta_{p}^{\delta}(u)=\operatorname{div}\left[\phi_{\delta}(|D u|) D u\right]
$$


can be written as

$$
\Delta_{p}^{\delta}(u)=\phi_{\delta}(|D u|) E_{i j}^{\delta} D_{i j} u
$$

where

$$
E_{i j}^{\delta}=E_{i j}^{\delta}(D u)=\left(\delta_{i j}-d_{i} d_{j}\right)+\sigma_{\delta}(|D u|) d_{i} d_{j}
$$

In particular, we have

$$
\Delta_{p}^{\delta}(u)=\Delta_{p}(u) \text { for }|D u| \geq \delta,
$$

and

$$
\Delta_{p}^{\delta}(u)=\phi_{\delta}(0) \Delta u \text { for }|D u| \leq \delta / 2 .
$$

According to (2.12) and the properties of $\phi_{\delta}$, we define $H_{\delta}(X)$ by

$$
H_{\delta}(X)=H(X)+(\lambda / 2) \delta^{p-1}|X|^{2}, \quad \text { for } X \in \mathbf{R}^{N} \text {. }
$$

b. Next we solve $\left(\mathrm{P}_{\varepsilon, \delta}\right)$ for smooth initial data. Theorems VI.4.1 and V.8.1 in [LSU] give

Lemma 2.4. Fix $\varepsilon, \delta>0$ and let $H_{\delta}$ be as in (2.13). Let $u_{0}(x)$ be $C^{\infty}\left(\mathbf{R}^{N}\right)$ and bounded, with bounded derivatives of all orders. Then, there exists a unique $u=u_{\varepsilon, \delta}(x, t)$ solution of $\left(\mathbf{P}_{\varepsilon, \delta}\right)$, which is $C^{\infty}\left(\mathbf{R}^{N} \times(0, \infty)\right)$ and bounded, together with its derivatives of all orders. In particular

$$
\sup _{\mathbf{R}^{N} \times(0, \infty)}|u(x, t)| \leq M_{0}=\sup _{\mathbf{R}^{N}}\left|u_{0}\right|
$$

and

$$
\sup _{\mathbf{R}^{N} \times(0, \infty)}|D u(x, t)| \leq M_{1},
$$

where $M_{1}$ depends on $\varepsilon, \delta, M_{0}$, and $\sup _{\mathbf{R}^{N}}\left|D u_{0}\right|$.

c. Now we can prove

Proposition 2.5. Let $u_{0}(x)$ and $u=u_{\varepsilon, \delta}(x, t)$ be as in Lemma 2.4. Then $\Delta_{p}^{\delta}(u) \leq k / t$ holds in $\mathbf{R}^{N} \times(0, \infty)$.

Proof of Proposition 2.5. As explained above, we first consider the linear operator

$$
\mathscr{L}_{\delta}(\varphi)=\varphi_{t}-\varepsilon D_{i}\left[\phi_{\delta}(|D u|) E_{i j}^{\delta}(D u) D_{j} \varphi\right]+H_{\delta}^{k}(D u) D_{k} \varphi,
$$

then show that $\theta=\Delta_{p}^{\delta}(u)$ satisfies

$$
\mathscr{L}_{\delta}(\theta) \leq-\theta^{2} / k
$$

and finally, we apply the Maximum Principle (e.g., as stated in [IKO, Theorem 8]).

Let us obtain (2.15). Set $\mathscr{E}_{\delta}=\left(E_{i j}^{\delta}\right)$, and $Q, B^{2}, T$, and $A$ as in (2.10). We have

$$
\begin{aligned}
\theta & =\Delta_{p}^{\delta}(u)=\phi_{\delta}(|D u|) \operatorname{Trace}\left(\mathscr{E}_{\delta} D^{2} u\right) \\
& =\phi_{\delta}(|D u|)\left[(T-A)+\sigma_{\delta}(|D u|) A\right]
\end{aligned}
$$

and

$$
\theta^{2} / N \leq \phi_{\delta}(|D u|)^{2}\left[\left(Q+A^{2}-2 B^{2}\right)+\sigma_{\delta}(|D u|)^{2} A^{2}\right]
$$


as in Lemma 2.1. On the other hand (set $\left.\mathscr{H}_{\delta}=\left(H_{\delta}^{i j}\right)\right)$,

$$
\begin{aligned}
\mathscr{L}_{\delta}(\theta) & =-\phi_{\delta}(|D u|) \operatorname{Trace}\left[\left(\mathscr{L}_{\delta} D^{2} u\right)\left(\mathscr{E}_{\delta} D^{2} u\right)\right] \\
& \leq-\lambda\left(|D u|^{p-1}+\delta^{p-1}\right) \phi_{\delta}(|D u|)\left\{\sigma_{\delta}(|D u|) B^{2}+\left[Q+A^{2}-2 B^{2}\right]+\left[B^{2}-A^{2}\right]\right\} .
\end{aligned}
$$

Since $\phi_{\delta}(|D u|) \leq|D u|^{p-1}+\delta^{p-1}$ and $1 \leq \sigma_{\delta} \leq p$, we obtain

$$
\mathscr{L}_{\delta}(\theta) \leq-\frac{\lambda}{p} \phi_{\delta}(|D u|)^{2}\left\{\left[Q+A^{2}-2 B^{2}\right]+\sigma_{\delta}(|D u|)^{2} A^{2}\right\} \leq-\frac{\lambda}{p N} \theta^{2},
$$

where we have used $A^{2} \leq B^{2}$ and (2.16).

To complete the proof of Theorem 2 we have yet to let $\delta$ tend to 0 in Proposition 2.5. This will be done in $\S 5$, where we will study the limits as $\delta \rightarrow 0$ and as $\varepsilon \rightarrow 0$ of the solutions and the various terms in $\left(\mathrm{P}_{\varepsilon, \delta}\right)$ and $\left(\mathbf{P}_{\varepsilon}\right)$, respectively.

Remark 2.6. Actually we can consider an alternative nonlinear vanishing viscosity related to the convex hamiltonian $H$, namely by replacing the $p$-Laplacian with the nonlinear operator $\operatorname{div} D H(D u)$. In this case, we can avoid the use of Lemma 2.1 and by following the same procedure used in [L] for the usual vanishing viscosity (see also 1d), we obtain a new semiconcavity inequality of the form

$$
\operatorname{div} D H(D u) \leq N / t .
$$

\section{GRADiEnT ESTimates}

In this section, we obtain two different local estimates for the gradient of the solutions $u_{\varepsilon}$ and $u$ of problems $\left(\mathrm{P}_{\varepsilon}\right)$ and $(\mathrm{P})$.

For fixed $R>0$ and $0<\tau<T$ we set $Q_{R, \tau}=B_{R}(0) \times(\tau, T]$. In the first estimate, we establish a bound of $\left|D u_{\varepsilon}\right|$ over $Q_{R, \tau}$ in terms of the oscillation of $u_{\varepsilon}$ over a larger region, say $Q_{2 R, \tau / 2}$. To be more precise, we consider a cut-off function $\zeta \in C^{\infty}\left(Q_{R, \tau}\right), 0 \leq \zeta(x, t) \leq 1$, and such that $\zeta(x, t)=0$ on the parabolic boundary of $Q_{R, \tau}$. We also define

$$
\omega_{R, \tau}=\frac{\sup }{Q_{R, \tau}} f(t, u)-\frac{\inf }{Q_{R, \tau}} f(t, u),
$$

where the function $f(t, u)$ is given by

$$
f(t, u)=u+\varepsilon k(p-1) \log (t / \tau),
$$

where $p>1$ (and $k=p N / \lambda$ is the constant in Theorem 1 ).

Theorem 2. Let $u=u_{\varepsilon}(x, t)$ be the solution of $\left(\mathrm{P}_{\varepsilon}\right)$. For $(x, t) \in \overline{Q_{R}, \tau}$, we have

$$
\zeta(x, t)|D u(x, t)| \leq C\left\{\left(\varepsilon+\|D \zeta\|_{\infty}\right) \omega_{R, \tau}+\left[\left(\left\|\zeta_{t}\right\|_{\infty}+\varepsilon\|D \zeta\|_{\infty}^{2 p}\right) \omega_{R, \tau}\right]^{1 /(p+1)}\right\},
$$

where the constant $C$ depends on $p, N, \lambda$, and $\Lambda$.

Remark. Theorem 3 will be proved by using the classical Bernstein method. As explained in the Introduction, the semiconcavity estimate in Theorem 2 and the definition of $f(t, u)$ in (3.1) will play a key role in obtaining estimates from the linear operator $\mathscr{L}_{\delta}$ in (2.14) (see Lemma 3.3 and inequality (3.17) below).

By letting $\varepsilon \rightarrow 0$ in Theorem 3, we obtain 
Corollary 1. Let $u=u(x, t)$ be the unique viscosity solution of $(\mathrm{P})$, and let $\zeta(x, t)$ be as above. We have

$$
\zeta(x, t)|D u(x, t)| \leq C\left\{\|D \zeta\|_{\infty} \omega_{R, \tau}(u)+\left[\left\|\zeta_{t}\right\|_{\infty} \omega_{R, \tau}(u)\right]^{1 /(p+1)}\right\}
$$

where $\omega_{R, \tau}(u)$ stands for the oscillation of $u$ over $\overline{Q_{R}, \tau}$.

The second type of gradient estimate that we prove in this section is a bound of $\left|D u_{\varepsilon}\right|$ in a strip $\mathbf{R}^{N} \times[0, T)$, for some $0<T \leq+\infty$, in terms of the behaviour of $\left|D u_{0}(x)\right|$ as $|x| \rightarrow \infty$. We have

Theorem 3. Let $u=u_{\varepsilon}(x, t)$ be the solution of $\left(\mathbf{P}_{\varepsilon}\right)$ with initial data $u_{0}(x)$ such that

$$
K_{0}=\sup _{x \in \mathbf{R}^{N}}(1+|x|)^{-1 / p}\left|D u_{0}(x)\right|<+\infty .
$$

Then, there exists $0<T_{\varepsilon}<+\infty$ such that

$$
(1+|x|)^{-1 / p}|D u(x, t)| \leq C\left[\left(e^{\varepsilon \mu\left(T_{\varepsilon}-t\right)}-1\right) / \varepsilon\right]^{-1 / p},
$$

holds a.e. in $\mathbf{R}^{N} \times\left[0, T_{\varepsilon}\right)$. The constant $C$ depends on $p$ and $\Lambda$, while $\mu$ depends only on $p$.

Remark. The finite blow-up time $T_{\varepsilon}$ of the estimate above is given by

$$
T_{\varepsilon}=\frac{1}{\varepsilon \mu} \log \left(1+\frac{\varepsilon K_{0}^{-p}}{\Lambda+\varepsilon}\right) .
$$

The corresponding estimate for the solution of problem $(P)$ is obtained by letting $\varepsilon \rightarrow 0$ in Theorem 3. We have

Corollary 2. Let $u=u(x, t)$ be the unique solution of $(\mathrm{P})$ and let $u_{0}(x)$ be as in Theorem 3. Then

$$
|D u(x, t)|^{p} \leq C(1+|x|) /(T-t)
$$

holds a.e. in $\mathbf{R}^{N} \times[0, T)$. $T$ is given by $T=C_{p, \Lambda} K_{0}^{-p}$.

Let us prove now Theorem 2.

Proof of Theorem 2. As in the proof of Theorem 1, we first deal with $u=$ $u_{\varepsilon, \delta}(x, t)$ as in Lemma 2.4 and then pass to the limit as $\delta \rightarrow 0$.

We estimate the quantity

$$
S=S_{R, \tau}=\frac{\max }{Q_{R, \tau}} \zeta^{p+1}|D u|^{p+1}
$$

with the aid of the function

$$
Z(x, t)=\zeta^{p+1}|D u|^{p+1}-\mu f(t, u),
$$

where the constant $\mu$ is defined by

$$
\mu=(c+S) / 2\left(c+\omega_{R, \tau}\right) \quad(0<c<1) .
$$

Let $\left(x_{0}, t_{0}\right) \in \overline{Q_{R}, \tau}$ be such that

$$
Z\left(x_{0}, t_{0}\right)=\frac{\max }{Q_{R, \tau}} Z(x, t) .
$$

Now we distinguish several cases. 
If either $\left|x_{0}\right|=R$ or $t_{0}=\tau$ then $\zeta\left(x_{0}, t_{0}\right)=0$ and

$$
Z(x, t) \leq Z\left(x_{0}, t_{0}\right)=-\mu f\left(t_{0}, u\left(x_{0}, t_{0}\right)\right) \quad \text { for }(x, t) \in \overline{Q_{R, \tau}},
$$

which implies

$$
S \leq \omega_{R, \tau}(c+S) / 2\left(c+\omega_{R, \tau}\right),
$$

and therefore

$$
S \leq c .
$$

If on the contrary $\left|x_{0}\right|<R$ and $\tau<t_{0} \leq T$, then we have two possibilities. If $\left|D u\left(x_{0}, t_{0}\right)\right| \leq \delta$, then

$$
Z(x, t) \leq Z\left(x_{0}, t_{0}\right) \leq \delta^{p+1}-\mu f\left(t_{0}, u\left(x_{0}, t_{0}\right)\right)
$$

and (3.5) imply

$$
S \leq 2 \delta^{p+1}+c .
$$

In the remaining case $\left|D u\left(x_{0}, t_{0}\right)\right|>\delta$, we argue as follows.

We consider the linear operator $\mathscr{L}_{\delta}$ in (2.14), which in the range $|D u|>\delta$ reads

$$
\mathscr{L}_{\delta}(\varphi)=\varphi_{t}-\varepsilon D_{i}\left[|D u|^{p-1} E_{i j}(D u) D_{j} \varphi\right]+H^{k}(D u) D_{k} \varphi,
$$

thanks to our construction of $\phi_{\delta}$ in Lemma 2.2(i). By writing $\mathscr{L}_{\delta}(\varphi)$ in nondivergence form, we obtain

$$
\mathscr{L}_{\delta}(\varphi)=\varphi_{t}-\varepsilon|D u|^{p-1} E_{i j}(D u) D_{i j} \varphi+\left[H_{\delta}^{k}(D u)-\varepsilon B_{k}(D u)\right] D_{k} \varphi,
$$

for $|D u|>\delta$, where the coefficients $B_{k}(D u)$ are given by

$$
\begin{aligned}
B_{k} & =D_{i}\left[|D u|^{p-1} E_{i k}(D u)\right] \\
& =2(p-1)|D u|^{p-3}\left[D_{i} u D_{i k} u-A D_{k} u\right]+(p-1) \frac{D_{k} u}{|D u|^{2}} \Delta_{p}(u),
\end{aligned}
$$

and $A$ was defined in (2.10).

We will prove

Proposition 3.2. At the points $(x, t) \in Q_{R, \tau}$ where $|D u(x, t)|>\delta$, we have

$$
\begin{aligned}
\mathscr{L}_{\delta}(Z) \leq & -\frac{\lambda}{p+1} \mu|D u|^{p+1}-C \varepsilon \zeta^{p+1} \Delta_{p}(u)^{2} \\
& +C\left[\left\|\zeta_{t}\right\|_{\infty}+\varepsilon\|D \zeta\|_{\infty}^{2} \zeta^{p-1}|D u|^{p-1}+\|D \zeta\|_{\infty} \zeta^{p}|D u|^{p}\right]|D u|^{p+1}
\end{aligned}
$$

where $C=C(p, N, \lambda, \Lambda)>0$.

We postpone the proof of Proposition 3.2 and continue with the estimate of $S$ in the case $\left|D u\left(x_{0}, t_{0}\right)\right|>\delta$.

Since $\left(x_{0}, t_{0}\right) \in Q_{R, \tau}$ is a point of maximum of $Z(x, t)$, we have $0 \leq$ $\mathscr{L}_{\delta}(Z)\left(x_{0}, t_{0}\right)$. This inequality, Proposition 3.2 and the fact that $D u\left(x_{0}, t_{0}\right) \neq$ 0 imply

$$
\mu \leq C\left[\left\|\zeta_{t}\right\|_{\infty}+\varepsilon\|D \zeta\|_{\infty}^{2} \zeta^{p-1}|D u|^{p-1}+\|D \zeta\|_{\infty} \zeta^{p}|D u|^{p}\right] .
$$

From our definition of $\mu$ in (3.5) and Hölder's inequality, we obtain

$$
\frac{1}{c+\omega_{R, \tau}} S \leq C\left[\left\|\zeta_{t}\right\|_{\infty}+\varepsilon\|D \zeta\|_{\infty}^{2 p}+\left(\varepsilon+\|D \zeta\|_{\infty}\right) S^{p /(p+1)}\right],
$$


hence

$$
S^{p /(p+1)}\left[\frac{1}{c+\omega_{R, \tau}} S^{1 /(p+1)}-C\left(\varepsilon+\|D \zeta\|_{\infty}\right)\right] \leq C\left(\left\|\zeta_{t}\right\|_{\infty}+\varepsilon\|D \zeta\|_{\infty}^{2 p}\right) .
$$

For $\alpha>0$ to be chosen, we now face two different possibilities: Either

$$
S^{1 /(p+1)} /\left(c+\omega_{R, \tau}\right)-C\left(\varepsilon+\|D \zeta\|_{\infty}\right) \leq \alpha,
$$

in which case

$$
S \leq\left[\left(c+\omega_{R, \tau}\right)\left(\alpha+C\left(\varepsilon+\|D \zeta\|_{\infty}\right)\right)\right]^{p+1},
$$

or

$$
S^{1 /(p+1)} /\left(c+\omega_{R, \tau}\right)-C\left(\varepsilon+\|D \zeta\|_{\infty}\right) \geq \alpha
$$

which gives

$$
S \leq\left[(C / \alpha)\left(\left\|\zeta_{t}\right\|_{\infty}+\varepsilon\|D \zeta\|_{\infty}^{2 p}\right)\right]^{(p+1) / p}
$$

From (3.9), (3.10), and the definition of $S$ in (3.3), we obtain $\zeta(x, t)|D u(x, t)| \leq\left(c+\omega_{R, \tau}\right)\left(\alpha+C\left(\varepsilon+\|D \zeta\|_{\infty}\right)\right)+\left[(C / \alpha)\left(\left\|\zeta_{t}\right\|_{\infty}+\varepsilon\|D \zeta\|_{\infty}^{2 p}\right)\right]^{1 / p}$, for all $(x, t) \in \overline{Q_{R} \tau}$. Now we minimize with respect to $\alpha$ the right side of this expression. The result is

$$
\begin{aligned}
\zeta(x, t)|D u(x, t)| \leq C\{( & \left.+\|D \zeta\|_{\infty}\right)\left(c+\omega_{R, \tau}\right) \\
& \left.+\left[\left(\left\|\zeta_{t}\right\|_{\infty}+\varepsilon\|D \zeta\|_{\infty}^{2 p}\right)\left(c+\omega_{R, \tau}\right)\right]^{1 /(p+1)}\right\},
\end{aligned}
$$

for $(x, t) \in \overline{Q_{R}, \tau}$. Finally, Theorem 2 follows by letting $c$ and $\delta$ tend to 0 in (3.6), (3.7), and (3.11).

To prove Proposition 3.2, we first compute $\mathscr{L}_{\delta}(Z)$.

Lemma 3.3. We have

$$
\begin{aligned}
\mathscr{L}_{\delta}(Z)= & -\mu\left[H_{\delta}^{j}(D u) D_{j} u-H_{\delta}(D u)\right]+\mu\left[\varepsilon(p-1) \Delta_{p}(u)-f_{t}\right] \\
& -\varepsilon(p+1) \zeta^{p+1} D_{i}\left[|D u|^{p-1} D_{j} u\right] D_{j}\left[|D u|^{p-1} D_{i} u\right] \\
& -2 \varepsilon(p+1) D_{j}\left(\zeta^{p+1}\right)|D u|^{p-1} D_{i} u D_{i}\left[|D u|^{p-1} D_{j} u\right] \\
& +\mathscr{L}_{\delta}\left(\zeta^{p+1}\right)|D u|^{p+1}
\end{aligned}
$$

at the points $(x, t) \in Q_{R, \tau}$ where $|D u(x, t)|>\delta$.

Proof of Lemma 3.3. From the definition of $Z(x, t)$ in (3.4) we have

$$
\begin{aligned}
Z_{t}= & (p+1) \zeta^{p+1}|D u|^{p-1} D_{j} u\left[\varepsilon D_{j} \Delta_{p}(u)-H_{\delta}^{k} D_{j k} u\right] \\
& +\left(\zeta^{p+1}\right)_{t}|D u|^{p+1}-\mu\left[f_{t}+\varepsilon \Delta_{p}(u)-H_{\delta}\right] .
\end{aligned}
$$

On the other hand, we get from (2.4) that

$$
\begin{aligned}
D_{j} u D_{j} \Delta_{p}(u) & =D_{j} u D_{i}\left[|D u|^{p-1} E_{i k} D_{j k} u\right] \\
& =D_{j} u|D u|^{p-1} E_{i k} D_{i j k} u+D_{j} u D_{j k} u B_{k} .
\end{aligned}
$$

By introducing the quantities defined in (2.10), this can be written as

$$
\begin{aligned}
D_{j} u D_{j} \Delta_{p}(u)= & D_{j} u|D u|^{p-1} E_{i k} D_{i j k} u+2(p-1)|D u|^{p-1}\left[B^{2}-A^{2}\right] \\
& +(p-1) A \Delta_{p}(u) .
\end{aligned}
$$


Now we substitute this identity in (3.13) and obtain

$$
\begin{aligned}
Z_{t}= & \varepsilon I_{1}+2 \varepsilon I_{2}+\varepsilon I_{3}-\mu\left[f_{t}+\varepsilon \Delta_{p}(u)-H_{\delta}\right] \\
& -\zeta^{p+1} H_{\delta}^{k}(D u) D_{k}\left[|D u|^{p+1}\right]+\left(\zeta^{p+1}\right)_{t}|D u|^{p+1},
\end{aligned}
$$

where

$$
\begin{aligned}
& I_{1}=(p+1) \zeta^{p+1}|D u|^{2(p-1)} D_{j} u E_{i k}(D u) D_{i j k} u, \\
& I_{2}=(p+1)(p-1) \zeta^{p+1}|D u|^{2(p-1)}\left[B^{2}-A^{2}\right], \\
& I_{2}=(p+1)(p-1) \zeta^{p+1}|D u|^{p-1} A \Delta_{p}(u) .
\end{aligned}
$$

Let us compute $D_{j} Z$ and $D_{i j} Z$. We have

$$
D_{j} Z=(p+1) \zeta^{p+1}|D u|^{p-1} D_{k} u D_{j k} u+D_{j}\left(\zeta^{p+1}\right)|D u|^{p+1}-\mu D_{j} u,
$$

and

$$
\begin{aligned}
D_{i j} Z= & (p+1) \zeta^{p+1}|D u|^{p-1} D_{k} u D_{i j k} u \\
& +(p+1) \zeta^{p+1} D_{j k} u D_{i}\left[|D u|^{p-1} D_{k} u\right] \\
& +2(p+1) D_{j}\left(\zeta^{p+1}\right)|D u|^{p-1} D_{k} u D_{i k} u+|D u|^{p+1} D_{i j}\left(\zeta^{p+1}\right)-\mu D_{i j} u .
\end{aligned}
$$

The terms $|D u|^{p-1} E_{i j}(D u) D_{i j} Z$ and $\left[H_{\delta}^{k}(D u)-\varepsilon B_{k}(D u)\right] Z_{k}$ in $\mathscr{L}_{\delta}(Z)$ are respectively

$$
\begin{aligned}
|D u|^{p-1} E_{i j} D_{i j} Z= & I_{1}-\mu \Delta_{p}(u) \\
& +(p+1) \zeta^{p+1} D_{i}\left[|D u|^{p-1} D_{j} u\right] D_{j}\left[|D u|^{p-1} D_{i} u\right] \\
& +2(p+1) D_{i}\left(\zeta^{p+1}\right)|D u|^{p-1} D_{j} u D_{j}\left[|D u|^{p-1} D_{i} u\right] \\
& +|D u|^{p+1}|D u|^{p-1} E_{i j}(D u) D_{i j}\left(\zeta^{p+1}\right),
\end{aligned}
$$

and

$$
\begin{aligned}
{\left[H_{\delta}^{k}(D u)-\varepsilon B_{k}(D u)\right] Z_{k}=} & \zeta^{p+1} H_{\delta}^{k}(D u) D_{k}\left[|D u|^{p+1}\right]-\mu H_{\delta}^{k}(D u) D_{k} u \\
& +|D u|^{p+1}\left[H_{\delta}^{k}-\varepsilon B_{k}\right] D_{k}\left(\zeta^{p+1}\right) \\
& -2 \varepsilon I_{2}-\varepsilon I_{3}+\varepsilon \mu(p-1) \Delta_{p}(u) .
\end{aligned}
$$

Putting together the expressions (3.14)-(3.16) yields (3.12).

Proof of Proposition 3.2. We estimate the different terms in (3.12).

(a) We use the hypotheses (2.1) on $H$ to obtain

$$
-\mu\left[H_{\delta}^{j}(D u) D_{j} u-H_{\delta}(D u)\right] \leq-\lambda \mu|D u|^{p+1} /(p+1) .
$$

(b) Thanks to Theorem 1 and our definition of $f(t, u)$ in (3.1) we have

$$
\varepsilon(p-1) \Delta_{p}(u)-f_{t} \leq 0 .
$$

(c) As to the last term in (3.12), we have

$$
\begin{gathered}
\mathscr{L}_{\delta}\left(\zeta^{p+1}\right)|D u|^{p+1} \leq C\left\{\left\|\zeta_{t}\right\|_{\infty}+\varepsilon\|D \zeta\|_{\infty}^{2} \zeta^{p-1}|D u|^{p-1}\right. \\
\left.+\|D \zeta\|_{\infty} \zeta^{p}|D u|^{p}\right\}|D u|^{p+1} \\
-\varepsilon(p+1) \zeta^{p} B_{k}(D u) D_{k} \zeta|D u|^{p+1}
\end{gathered}
$$

where we have used the right inequality in (2.1), and we have assumed that $\zeta$ satisfies $\left\|\zeta D^{2} \zeta\right\|_{\infty} \leq C\|D \zeta\|_{\infty}^{2}$. 
(d) Finally, the term

$$
\begin{aligned}
& -\varepsilon(p+1) \zeta^{p+1} D_{i}\left[|D u|^{p-1} D_{j} u\right] D_{j}\left[|D u|^{p-1} D_{i} u\right] \\
& \quad=-\varepsilon(p+1) \zeta^{p+1}|D u|^{2(p-1)} \operatorname{Trace}\left[\left(\mathscr{E} D^{2} u\right)^{2}\right],
\end{aligned}
$$

controls the fourth term in (3.12) and the last one in (3.18). Actually, the sum of all of these three terms can be estimated as

$$
\leq-\varepsilon((p+1) / 2 N) \zeta^{p+1} \Delta_{p}(u)^{2}+\varepsilon C(p, N)\|D \zeta\|_{\infty}^{2} \zeta^{p-1}|D u|^{p-1}|D u|^{p+1},
$$

by using Cauchy-Schwarz inequality and Lemma 2.1(b).

The proof of Theorem 2 being completed, we next prove Theorem 3 .

Proof of Theorem 3. We first proceed formally. Later on, we will indicate how the formal arguments can be made rigourous.

We consider the functions $\psi(x)=\left(1+|x|^{2}\right)^{-(p+1) / 2 p}$ and

$$
Z(x, t)=\psi(x)|D u(x, t)|^{p+1} .
$$

Theorem 3 will follow from the Maximum Principle, when applied to $Z(x, t)$ and the linear operator

$$
\mathscr{L}^{\prime}(\varphi)=\mathscr{L}(\varphi)+2 \varepsilon \psi(x)^{-1}|D u|^{p-1} E_{i j}(D u) D_{j} \psi(x) D_{i} \varphi,
$$

where $\mathscr{L}(\varphi)$ is as in (3.8), i.e.,

$$
\mathscr{L}(\varphi)=\varphi_{t}-\varepsilon|D u|^{p-1} E_{i j}(D u) D_{i j} \varphi+\left[H_{\delta}^{k}(D u)-\varepsilon B_{k}(D u)\right] D_{k} \varphi .
$$

From Lemma 3.3, we have

$$
\mathscr{L}\left(|D u|^{p+1}\right)=-\varepsilon(p+1)|D u|^{2(p-1)} \operatorname{Trace}\left[\left(\mathscr{E} D^{2} u\right)^{2}\right] .
$$

Straightforward computations lead to

$$
\mathscr{L}^{\prime}(Z)=\psi(x) \mathscr{L}\left(|D u|^{p+1}\right)
$$

$$
\begin{aligned}
& +\psi(x)^{-1}\left[H^{k}(D u)-\varepsilon B_{k}(D u)\right] D_{k} \psi(x) Z \\
& +\varepsilon \psi(x)^{-2}|D u|^{p-1} E_{i j}(D u)\left[2 D_{i} \psi(x) D_{j} \psi(x)-\psi(x) D_{i j} \psi(x)\right] Z .
\end{aligned}
$$
get

$$
\psi(x)^{-1} H^{k}(D u) D_{k} \psi(x) Z \leq \Lambda \psi(x)^{-\alpha}|D \psi(x)| Z^{\alpha},
$$

where $\alpha=(2 p+1) /(p+1)$. The last term in (3.19) is estimated as

$$
\leq \varepsilon C \psi(x)^{-\gamma}\left(|D \psi(x)|^{2}+\psi(x)\left|D^{2} \psi(x)\right|\right) Z^{\beta},
$$

where $\beta=2 p /(p+1)<\alpha$ and $\gamma=(3 p+1) /(p+1)$.

On the other hand, this last quantity is also an upper bound for the terms

$$
\psi(x) \mathscr{L}\left(|D u|^{p+1}\right)-\varepsilon \psi(x)^{-1} B_{k}(D u) D_{k} \psi(x) Z
$$

in (3.19) (again, we use Cauchy-Schwarz and Lemma 2.1(b)).

The coefficients of $Z^{\alpha}$ and $Z^{\beta}$ in (3.20) and (3.21) being uniformly bounded for $x \in \mathbf{R}^{N}$, we are led to the inequalities

$$
\mathscr{L}^{\prime}(Z) \leq C_{p}\left[\Lambda Z^{\alpha}+\varepsilon Z^{\beta}\right] \leq C_{p}\left[(\Lambda+\varepsilon) Z^{\alpha}+\varepsilon Z\right],
$$

for some $C_{p}>0$ depending on $p$. 
Finally, since

$$
\varphi(t)=\left\{(\Lambda+\varepsilon)\left[\left(e^{\varepsilon \mu\left(T_{e}-t\right)}-1\right) / \varepsilon\right]\right\}^{-(p+1) / p},
$$

(with $\mu=p C_{p} /(p+1)$ and $T_{\varepsilon}$ as in (3.2)) satisfies $\varphi^{\prime}=C_{p}\left[(\Lambda+\varepsilon) \varphi^{\alpha}+\varepsilon \varphi\right]$ and $\varphi(0)=K_{0}^{p+1} \geq Z(x, 0)$, the Maximum Principle implies $Z(x, t) \leq \varphi(t)$ in $\mathbf{R}^{N} \times\left[0, T_{\varepsilon}\right)$.

The arguments above can be put into rigourous form as follows. Consider the function

$$
Z_{\delta}(x, t)=\psi(x) \Phi_{\delta}(|D u|),
$$

where $u=u_{\varepsilon, \delta}(x, t)$ is as in Lemma 2.4 , and $\Phi_{\delta}=\Phi_{\delta}(r)$ is defined by $\Phi_{\delta}^{\prime}(r)=r \phi_{\delta}(r)$ for $r \geq 0$ and $\Phi_{\delta}(0)=0$. In particular, this gives

$$
r \Phi_{\delta}^{\prime \prime}(r) / \Phi_{\delta}^{\prime}(r)=1+r \phi_{\delta}^{\prime}(r) / \phi_{\delta}(r)=\sigma_{\delta}(r)
$$

(notations as in Lemma 2.2), and

$$
\mathscr{L}_{\delta}\left(Z_{\delta}\right)=-\varepsilon \phi_{\delta}(|D u|)^{2} \operatorname{Trace}\left[\left(\mathscr{E}_{\delta} D^{2} u\right)^{2}\right],
$$

where $\mathscr{L}_{\delta}$ comes from (2.14). The Maximum Principle is then applied to $Z_{\delta}$ and the linear operator

$$
\mathscr{L}_{\delta}^{\prime}(\varphi)=\mathscr{L}_{\delta}(\varphi)+2 \varepsilon \psi(x)^{-1} \phi_{\delta}(|D u|) E_{i j}^{\delta}(D u) D_{j} \psi(x) D_{i} \varphi .
$$

The details are left to the reader.

\section{Local estimates OF THE SOLUTIONS}

In this section, we construct some upper and lower local estimates for the solution $u_{\varepsilon}$ of problem $\left(\mathrm{P}_{\varepsilon}\right)$. Some of our considerations have been motivated by the explicit formulas in [L, BE] and by some ideas in [LSV]. 1 .

Our first result is a consequence of the semiconcavity estimate in Theorem

Proposition 4.1. Let $u_{\varepsilon}(x, t)$ be the solution of $\left(\mathrm{P}_{\varepsilon}\right)$. Then

$$
u_{\varepsilon}(x, t) \leq u_{\varepsilon}(y, \tau)+C_{p, \lambda} \frac{|x-y|^{1+1 / p}}{(t-\tau)^{1 / p}}+\varepsilon k \log \frac{t}{\tau},
$$

holds for every $x, y \in \mathbf{R}^{N}$ and $t>\tau>0$, where $k$ is the constant in Theorem 1 .

Before going into the proof of this proposition, we show another upper estimate of the solution, which holds down to $t=0$.

Lemma 4.2. Let $u_{0}(x)$ be a continuous function, and such that

$$
u_{0}(x) \leq A+B\left|x-x_{0}\right|^{1+1 / p}, \quad x \in \mathbf{R}^{N},
$$

for some $x_{0} \in \mathbf{R}^{N}, A \in \mathbf{R}$, and $B>0$. Then, the solution $u_{\varepsilon}(x, t)$ of $\left(\mathbf{P}_{\varepsilon}\right)$ with initial data $u_{0}(x)$ satisfies

$$
u_{\varepsilon}(x, t) \leq A+B\left|x-x_{0}\right|^{1+1 / p}\left(1+t / \tau_{0}\right)^{-1 / p}+\varepsilon k \log \left(1+t / \tau_{0}\right)
$$

for all $x \in \mathbf{R}^{N}$ and $t \geq 0$. The positive constant $\tau_{0}$ depends on $B$, $p$, and $\lambda$. Proof of Lemma 4.2. We recall the hypothesis (2.1) on $H(X)$, which gives

$$
H(X) \geq \lambda|X|^{p+1} / p(p+1) \text { for } X \in \mathbf{R}^{N},
$$


and consider the operator

$$
\mathscr{Q}_{\lambda}(\varphi)=\varphi_{t}+\lambda|D \varphi|^{p+1} / p(p+1)-\varepsilon \Delta_{p}(\varphi),
$$

together with

$$
\mathscr{Q}(\varphi)=\varphi_{t}+H(D \varphi)-\varepsilon \Delta_{p}(\varphi) .
$$

Let us compare $u_{\varepsilon}(x, t)$ to (we set $x_{0}=0$ for simplicity),

$$
U(x, t)=A+B|x|^{1+1 / p}\left(1+t / \tau_{0}\right)^{-1 / p}+\varepsilon k \log \left(1+t / \tau_{0}\right) .
$$

We have $0=\mathscr{Q}\left(u_{\varepsilon}\right) \geq \mathscr{Q}_{\lambda}\left(u_{\varepsilon}\right)$, as well as $\mathscr{Q}(U) \geq \mathscr{Q}_{\lambda}(U)$. On the other hand, $\mathscr{Q}_{\lambda}(U)$ can be made $\mathscr{Q}_{\lambda}(U)=0$ by choosing $\tau_{0}=\left(C_{p, \lambda} / B\right)^{p}$, where $C_{p, \lambda}=$ $(p /(p+1))(p / \lambda)^{1 / p}$. Since by (4.2) $U(x, 0) \geq u_{0}(x)$, the Maximum Principle yields $u_{\varepsilon}(x, t) \leq U(x, t)$.

Proof of Proposition 4.1. The solution $u_{\varepsilon}$ satisfies $\Delta_{p}\left(u_{\varepsilon}\right) \leq k / t$, hence

$$
u_{\varepsilon, t}+H\left(D u_{\varepsilon}\right)-\varepsilon k / t \leq 0 \text {. }
$$

Now, if $v(x, t)$ is the solution of the first order initial value problem: $v_{t}+$ $H(D v)=0$ in $\mathbf{R}^{N} \times[\tau,+\infty), v(x, \tau)=u_{\varepsilon}(x, \tau)$ in $\mathbf{R}^{N}$, we conclude that

$$
u_{\varepsilon}(x, t) \leq v(x, t)+\varepsilon k \log (t / \tau) \text { in } \mathbf{R}^{N}, \times[\tau,+\infty) .
$$

On the other hand, the Lax-Oleinik formula gives

$$
v(x, t)=\inf _{y \in \mathbf{R}^{N}}\left\{u_{\varepsilon}(y, \tau)+(t-\tau) H^{*}\left(\frac{x-y}{t-\tau}\right)\right\},
$$

where $H^{*}(X)=\sup _{Y \in \mathbf{R}^{N}}\{X Y-H(Y)\}$ is the polar function of $H(X)$. Finally, the hypothesis (2.1) on $H$ implies $H^{*}(X) \leq C_{p, \lambda}|X|^{1+1 / p}$ for all $X \in \mathbf{R}^{N}$, with $C_{p, \lambda}$ as in Lemma 4.2. Then (4.1) follows.

Remark. If the solution $u_{\varepsilon}(x, t)$ exists in a strip $\mathbf{R}^{N} \times[0, T), 0<T \leq+\infty$, we have from Proposition 4.1 that

$$
-\frac{C_{p, \lambda}}{(T-t)^{1 / p}} \leq \liminf _{|x| \rightarrow \infty} \frac{u_{\varepsilon}(x, t)}{|x|^{1+1 / p}} \leq \limsup _{|x| \rightarrow \infty} \frac{u_{\varepsilon}(x, t)}{|x|^{1+1 / p}} \leq \frac{C_{p}, \lambda}{t^{1 / p}},
$$

for $0<t<T$, and

$$
-\frac{C_{p, \lambda}}{T^{1 / p}} \leq \liminf _{|x| \rightarrow \infty} \frac{u_{0}(x)}{|x|^{1+1 / p}}
$$

The case $T=+\infty$ corresponds to

$$
0 \leq \liminf _{|x| \rightarrow \infty} \frac{u_{0}(x)}{|x|^{1+1 / p}}
$$

This condition is satisfied in the class of initial data that we consider in our next estimate.

Proposition 4.3. Assume that $u_{0}(x)$ is continuous and satisfies

$$
u_{0}(x) \geq-\left(a+b|x|^{r}\right) \quad \text { for all } x \in \mathbf{R}^{N},
$$

for some exponent

$$
0<r<1+1 / p
$$


and some $a, b>0$. Let $u_{\varepsilon}(x, t)$ be the solution of $\left(\mathbf{P}_{\varepsilon}\right)$ with initial data $u_{0}(x)$. For any $T>0$, we have

$$
u_{\varepsilon}(x, t) \geq-K-C_{p, \Lambda}|x|^{1+1 / p} /(T-t)^{1 / p}+\varepsilon k^{\prime} \log (1-t / T),
$$

for all $x, y \in \mathbf{R}^{N}$ and $0 \leq t<T$. The constant $K$ depends on $a, b, p$ and $r$, while $k^{\prime}=p N / \Lambda$.

Proof of Proposition 4.3. This time we obtain from (2.1) that

$$
H(X) \leq \Lambda|X|^{p+1} / p(p+1) \text { for } X \in \mathbf{R}^{N} .
$$

As in Lemma 4.2 we consider the operators $\mathscr{Q}$ and $\mathscr{Q}_{\Lambda}$, which now reads

$$
\mathscr{Q}_{\Lambda}(\varphi)=\varphi_{t}+\Lambda|D \varphi|^{p+1} / p(p+1)-\varepsilon \Delta_{p}(\varphi) .
$$

We have $\mathscr{Q}_{\Lambda}\left(u_{\varepsilon}\right) \geq \mathscr{Q}\left(u_{\varepsilon}\right)=0$ in $\mathbf{R}^{N} \times(0,+\infty)$. A subsolution for $\mathscr{Q}_{\Lambda}$ is constructed as follows.

Given $T>0$, we choose $c>0$ such that

$$
T_{c}=C_{p, \Lambda}^{p} c^{-p}, \quad C_{p, \Lambda}=(p /(p+1))(p / \Lambda)^{1 / p},
$$

satisfies $T_{c}>T$.

We use this $c$ and Hölder's inequality to obtain from (4.3) that

$$
u_{0}(x) \geq-\left(a+b|x|^{r}\right) \geq-\left[\left(a+b_{c}\right)+c|x|^{1+1 / p}\right],
$$

where $b_{c}=\left(b^{p+1} c^{-p r}\right)^{1 /((p+1)-p r)}$.

For $x \in \mathbf{R}^{N}$ and $0 \leq t<T_{c}$, we now define

$$
V(x, t)=-\left(a+b_{c}\right)-C(t)|x|^{1+1 / p}+\varepsilon f(t),
$$

with

$$
\begin{aligned}
C(t) & =C_{p, \Lambda}\left(T_{c}-t\right)^{-1 / p}, \\
f(t) & =k^{\prime} \log \left(1-t / T_{c}\right), \quad k^{\prime}=p N / \Lambda .
\end{aligned}
$$

Thanks to (4.4), we have $V(x, 0) \leq u_{0}(x)$ for $x \in \mathbf{R}^{N}$. On the other hand, $V(x, t)$ also satisfies

$$
\begin{aligned}
\mathscr{Q}_{\Lambda}(V)= & \varepsilon\left[f^{\prime}(t)+N((p+1) / p)^{p} C(t)^{p}\right] \\
& +\left[\left(\Lambda / p^{2}\right)((p+1) / p)^{p} C(t)^{p+1}-C^{\prime}(t)\right]|x|^{1+1 / p} \\
= & 0,
\end{aligned}
$$

thanks to the construction of $C(t)$ and $f(t)$. The assertion of the lemma then follows from the Maximum Principle.

\section{CONVERGENCE OF THE APPROXIMATE PROBLEMS}

In this section, we study the limits as $\delta \rightarrow 0$ and as $\varepsilon \rightarrow 0$ in problems $\left(\mathrm{P}_{\varepsilon, \delta}\right)$ and $\left(\mathrm{P}_{\varepsilon}\right)$, respectively. We establish some compactness properties of the nonlinear viscosity terms in both of the two problems. In the limits, we obtain the viscosity solutions of $\left(\mathrm{P}_{\varepsilon}\right)$ and $(\mathrm{P})$.

We first keep $\varepsilon>0$ fixed and let $\delta \rightarrow 0$ in problem $\left(\mathrm{P}_{\varepsilon, \delta}\right)$. Let us denote by $u_{\delta}=u_{\varepsilon, \delta}(x, t)$ the solution of this problem. We have 
Theorem 4. Let $\varepsilon>0$ be fixed. As $\delta \rightarrow 0$, the solutions $u_{\delta}(x, t)$ converge to a continuous function $u=u(x, t)$, uniformly on compact subsets of $\mathbf{R}^{N} \times$ $(0,+\infty)$. Moreover,

(a) For $i=1,2, \ldots, N$,

$$
\begin{aligned}
D_{i} u_{\delta}(x, t) \rightarrow & D u_{i}(x, t) \text { a.e. in } \mathbf{R}^{N} \times(0,+\infty), \text { and in } \\
& L_{\mathrm{loc}}^{r}\left(\mathbf{R}^{N} \times(0,+\infty)\right) \text { for every } p+1 \leq r<+\infty .
\end{aligned}
$$

(b) For fuxed $t>0, \Delta_{p}^{\delta} u_{\delta}(\cdot, t)$ converges to $\Delta_{p} u(\cdot, t)$ in the strong topology of $W_{\text {loc }}^{-1, s}\left(\mathbf{R}^{N}\right)$, for every $1<s<+\infty$. By this we mean that for any $\zeta \in C_{0}^{\infty}\left(\mathbf{R}^{N}\right)$,

$$
\zeta(\cdot) \Delta_{p}^{\delta} u_{\delta}(\cdot, t) \rightarrow \zeta(\cdot) \Delta_{p} u(\cdot, t) \quad \text { in } W^{-1, s}\left(\mathbf{R}^{N}\right) .
$$

Proof of Theorem 4. We divide the proof in several steps. In the first one, the semiconcavity estimate is used to show the convergence in (5.2).

Step 1. According to the results in [Mu], bounded sets of measures in $\mathbf{R}^{N}$ which are also bounded in $W^{-1, r}\left(\mathbf{R}^{N}\right)$, are relatively compact in $W_{\text {loc }}^{-1, s}\left(\mathbf{R}^{N}\right)$, for every $1<s<r<+\infty$.

Our estimates in $\S \S 3$ and 4 imply that $\left\{\left|D u_{\delta}\right|\right\}_{\delta>0}$ is uniformly bounded in any compact subset of $\mathbf{R}^{N} \times(0,+\infty)$. Therefore, for fixed $t>0$ and $\zeta \in C_{0}^{\infty}\left(\mathbf{R}^{N}\right)$, the set $\left\{\zeta(\cdot) \Delta_{p}^{\delta} u_{\delta}(\cdot, t)\right\}_{\delta>0}$ is uniformly bounded in $W^{-1, r}\left(\mathbf{R}^{N}\right)$, for every $1<r \leq+\infty$.

On the other hand, the semiconcavity $\Delta_{p}^{\delta} u_{\delta}(\cdot, t) \leq k / t$ implies that $\left\{\Delta_{p}^{\delta} u_{\delta}(\cdot, t)\right\}_{\delta>0}$ is a bounded set of measures in $\mathbf{R}^{N}$, by using standard arguments (cf., e.g., [Mu, Remarque 3]). Hence $\left\{\zeta(\cdot) \Delta_{p}^{\delta} u_{\delta}(\cdot, t)\right\}_{\delta>0}$ is also a bounded set of (compactly supported) measures.

Then, by Theorem 8 in [Mu], a subsequence of $\left\{\zeta(\cdot) \Delta_{p}^{\delta} u_{\delta}(\cdot, t)\right\}_{\delta>0}$ (that we will label also with the subscript $\delta$ ) converges to some measure $\mu_{t}$ in $W^{-1, s}\left(\mathbf{R}^{N}\right)$.

As to the function $u_{\delta}$ and its gradient $D u_{\delta}$, we have that as $\delta \rightarrow 0$ (again a subsequence), $u_{\delta} \rightarrow u$ in the weak- ${ }^{*}$ topology of $L_{\text {loc }}^{\infty}\left(\mathbf{R}^{N} \times(0,+\infty)\right)$, though for fixed $t>0, u_{\delta}(\cdot, t) \rightarrow u(\cdot, t)$ uniformly on compact subsets of $\mathbf{R}^{N}$. The convergence of $D u_{\delta} \rightarrow D u$ and of $\phi_{\delta}\left(\left|D u_{\delta}\right|\right) D u_{\delta}$ to some $V=V(x, t)$ also take place in the weak- ${ }^{*}$ topology of $L_{\text {loc }}^{\infty}\left(\mathbf{R}^{N} \times(0,+\infty)\right)$. Actually, one has $\mu_{t}=\zeta(\cdot) \operatorname{div} V(\cdot, t)$. Next we show that

$$
V(\cdot, t)=|D u(\cdot, t)|^{p-1} D u(\cdot, t) .
$$

To this aim, we use Minty's method and the strong convergence of the nonlinear viscosity term as follows.

By the monotonicity of the map $X \in \mathbf{R}^{N} \rightarrow \varphi_{\delta}(|X|) X$ we have that for every $\varphi \in W_{\mathrm{loc}}^{1, s^{\prime}}\left(\mathbf{R}^{N}\right)\left(1 / s+1 / s^{\prime}=1\right)$,

$$
\begin{aligned}
0 \leq & \int \zeta(x)^{2}\left[\phi_{\delta}\left(\left|D u_{\delta}\right|\right) D u_{\delta}-\phi_{\delta}(|D \varphi|) D \varphi\right]\left[D u_{\delta}(x, t)-D \varphi(x)\right] d x \\
= & -\left\langle\zeta(\cdot) \Delta_{p}^{\delta} u_{\delta}(\cdot, t)-\zeta(\cdot) \Delta_{p}^{\delta} \varphi(\cdot), \zeta(\cdot)\left(u_{\delta}(\cdot, t)-\varphi(\cdot)\right)\right\rangle \\
& -2 \int \zeta(x)\left(u_{\delta}(x, t)-\varphi(x)\right)\left[\phi_{\delta}\left(\left|D u_{\delta}\right|\right) D u_{\delta}(x, t)\right.
\end{aligned}
$$

$$
\left.-\phi_{\delta}(|D \varphi|) D \varphi(x)\right] D \zeta(x) d x
$$

where $\langle\cdot, \cdot\rangle$ denotes the duality pairing between $W^{-1, s}\left(\mathbf{R}^{N}\right)$ and $W^{1, s^{\prime}}\left(\mathbf{R}^{N}\right)$. 
As $\delta \rightarrow 0$, the first term above converges to

$$
\left.-\left\langle\zeta(\cdot) \operatorname{div} V(\cdot, t)-\zeta(\cdot) \Delta_{p}(\varphi(\cdot)), \zeta(\cdot)(u(\cdot, t)-\varphi)\right)\right\rangle,
$$

by the strong convergence of $\zeta(\cdot) \Delta_{p}^{\delta} u_{\delta}(\cdot, t)$ in $W^{-1, s}$, the uniform convergence of $\phi_{\delta}(|X|) X$ to $|X|^{p-1} X$ for $X \in \mathbf{R}^{N}$, and the weak convergence of $D u_{\delta}(\cdot, t)$ in $W_{\text {loc }}^{1, s^{\prime}}\left(\mathbf{R}^{N}\right)$. By similar reasons, the second term tends to

$$
-2 \int \zeta(x)(u(x, t)-\varphi(x))\left[V(x, t)-|D \varphi(x)|^{p-1} D \varphi(x)\right] D \zeta(x) d x .
$$

Therefore, we have obtained

$$
\begin{aligned}
0 \leq & -\left\langle\zeta(\cdot) \operatorname{div} V(\cdot, t)-\zeta(\cdot) \Delta_{p} \varphi(\cdot), \zeta(\cdot)(u(\cdot, t)-\varphi(\cdot))\right\rangle \\
& -2 \int \zeta(x)(u(x, t)-\varphi(x))\left[V(x, t)-|D \varphi(x)|^{p-1} D \varphi(x)\right] D \zeta(x) d x \\
= & \int \zeta(x)^{2}\left[V(x, t)-|D \varphi(x)|^{p-1} D \varphi(x)\right][D u(x, t)-D \varphi(x)] d x,
\end{aligned}
$$

which implies $V=|D u|^{p-1} D u$ by standard arguments.

Step 2. Now we show the convergence in (5.1). Our construction of $\phi_{\delta}$ in Lemma 2.2 preserves the coercivity properties of the map $X \in \mathbf{R}^{N} \rightarrow|X|^{p-1} X$. Actually, it can be easily checked that

$$
C_{p}|X-Y|^{p+1} \leq\left[\phi_{\delta}(|X|) X-\phi_{\delta}(|Y|) Y\right][X-Y]
$$

holds for all $X, Y \in \mathbf{R}^{N}$ and some positive constant $C_{p}$. We use this coercivity to get, for any fixed $t>0$,

$$
\begin{aligned}
C_{p} \int \zeta(x)^{2}\left|D u_{\delta}(x, t)-D u(x, t)\right|^{p+1} d x & \\
\leq & -\left\langle\zeta(\cdot) \Delta_{p}^{\delta} u_{\delta}(\cdot, t)-\zeta(\cdot) \Delta_{p}^{\delta} u(\cdot, t), \zeta(\cdot)\left(u_{\delta}(\cdot, t)-u(\cdot, t)\right)\right\rangle \\
& -2 \int \zeta(x)\left(u_{\delta}(x, t)-u(x, t)\right)\left[\phi_{\delta}\left(\left|D u_{\delta}\right|\right) D u_{\delta}(x, t)\right. \\
& \left.-\phi_{\delta}(|D u|) D u(x, t)\right] D \zeta(x) d x .
\end{aligned}
$$

As $\delta \rightarrow 0$, we obtain

$$
\int \zeta(x)^{2}\left|D u_{\delta}(x, t)-D u(x, t)\right|^{p+1} d x \rightarrow 0 .
$$

Fix now any $0<\tau<T<+\infty$. Since $\left\{\zeta\left|D u_{\delta}\right|\right\}_{\delta>0}$ is uniformly bounded in $\mathbf{R}^{N} \times[\tau, T]$, Lebesgue's dominated convergence theorem yields

$$
\lim _{\delta \rightarrow 0} \int_{\tau}^{T} \int_{\mathbf{R}^{N}} \zeta(x)^{2}\left|D u_{\delta}(x, t)-D u(x, t)\right|^{p+1} d x d t=0 .
$$

Step 3. In this step we prove the convergence $u_{\delta} \rightarrow u$ uniformly on compact subsets of $\mathbf{R}^{N} \times(0,+\infty)$. We already have that for any $t>0$,

$$
u_{\delta}(\cdot, t) \rightarrow u(\cdot, t) \quad \text { uniformly on compacts subsets of } \mathbf{R}^{N} \text {. }
$$

Let us fix $0<\tau<T, r^{\prime}>N$ and any open and bounded $\Omega \subset \mathbf{R}^{N}$, with $\operatorname{supp} \zeta \subset \Omega$.

Then $W_{0}^{1, r^{\prime}}(\Omega)$ is compactly embedded in $\mathscr{C}_{0}(\Omega)$ (the Banach space of functions which are continuous on $\bar{\Omega}$ and vanish on $\partial \Omega$ ). By duality $\mathscr{C}_{0}(\Omega)^{\prime}$ also 
embeds compactly in $W^{-1, r}(\Omega)$. A well-known interpolation inequality yields (with the usual $L^{2}(\Omega)$ identifications)

$$
\begin{gathered}
\max _{x \in \bar{\Omega}}\left|\zeta(x)\left(u_{\delta}\left(x, t_{1}\right)-u_{\delta}\left(x, t_{2}\right)\right)\right| \leq \eta \sup _{\tau \leq t \leq T}\left\|\zeta(\cdot) u_{\delta}(\cdot, t)\right\|_{W_{0}^{1, r^{\prime}}(\Omega)} \\
+C_{\eta}\left\|\zeta(\cdot)\left(u_{\delta}\left(\cdot, t_{1}\right)-u_{\delta}\left(\cdot, t_{2}\right)\right)\right\|_{W^{-1, r}(\Omega)}
\end{gathered}
$$

for $\tau \leq t_{1}<t_{2} \leq T$ and $\eta>0$ to be fixed small enough.

The bounds in $\S \S 3$ and 4 give that the coefficient of $\eta$ above is uniformly bounded. This fact and the equation

$$
u_{\delta, t}+H_{\delta}\left(D u_{\delta}\right)=\varepsilon \Delta_{p}^{\delta} u_{\delta} \quad \text { in } W_{\text {loc }}^{-1, r}\left(\mathbf{R}^{N} \times(0,+\infty)\right),
$$

imply

$$
\begin{aligned}
& \left\|\zeta(\cdot)\left(u_{\delta}\left(\cdot, t_{1}\right)-u_{\delta}\left(\cdot, t_{2}\right)\right)\right\|_{W^{-1, r}(\Omega)} \\
& \quad \leq\left|t_{1}-t_{2}\right| \sup _{\tau \leq t \leq T}\left\|\varepsilon \Delta_{p}^{\delta} u_{\delta}(\cdot, t)-H_{\delta}\left(D u_{\delta}(\cdot, t)\right)\right\|_{W^{-1, r}(\Omega)}
\end{aligned}
$$

hence

$$
\max _{x \in \bar{\Omega}}\left|\zeta(x)\left(u_{\delta}\left(x, t_{1}\right)-u_{\delta}\left(x, t_{2}\right)\right)\right|=o(1)
$$

as $\left|t_{1}-t_{2}\right| \rightarrow 0$.

Then it follows the convergence $u_{\delta} \rightarrow u$ uniformly on $\bar{\Omega} \times[\tau, T]$.

\section{REFERENCES}

[ALM] L. Alvarez, P. L. Lions, and J. M. Morel, Image selective smoothing and edge detection by nonlinear diffusion, SIAM J. Numer. Anal. 29 (1992), 845-866.

[A] D. G. Aronson, The porous medium equation, Noninear Diffusion Problems (A. Fasano and M. Primicerio, eds.), Lecture Notes in Math., vol. 1224, Springer-Verlag, Berlin and New York, 1986.

[BE] M. Bardi and L. C. Evans, On Hopf's formulas for solutions of Hamilton-Jacobi equations, Nonlinear Anal. 8 (1984), 1373-1381.

[B] G. Barles, $A$ weak Bernstein method for fully nonlinear elliptic equations, Differential Integral Equations 4 (1991), 241-262.

[BP] G. Barles and B. Perthame, Discontinuous solutions of deterministic optimal stopping time problems, Math. Model. Numer. Anal. 21 (1987), 557-579.

[CGG] Y. Chen, Y. Giga, and S. Goto, Uniqueness and existence of viscosity solutions of generalized mean curvature flow equations, J. Differential Geom. 33 (1991), 749-786.

[C] M. G. Crandall, Semidifferentials, quadratic forms and fully nonlinear elliptic equations of second order, Ann. Inst. H. Poincaré, Anal. Non Linéaire 6 (1989), 419-435.

[CEL] M. G. Crandall, L. C. Evans, and P. L. Lions, Some properties of viscosity solutions of Hamilton-Jacobi equations, Trans. Amer. Math. Soc. 282 (1984), 487-502.

[CIL] M. G. Crandall, H. Ishii, and P. L. Lions, User's guide to viscosity solutions of second order partial differential equations, Bull. Amer. Math. Soc. 27 (1992), 1-67.

[CLS] M. G. Crandall, P. L. Lions, and P. E. Souganidis, Maximal solutions and universal bounds for some partial differential equations of evolution, Arch. Rational Mech. Anal. 105 (1989), 163-190.

[EV1] J. R. Esteban and J. L. Vázquez, Homogeneous diffusion in $\mathbf{R}$ with power-like nonlinear diffusivity, Arch. Rational Mech. Anal. 103 (1988), 39-80.

[EV2] __ Régularité des solutions positives de l'équation parabolique p-laplacienne, C. R. Acad. Sci. Paris 310 (1990), 105-110.

[EV3] _ Regularity of solutions of nonlinear heat equations with power-like nonlinearities in several space dimensions, in preparation. 
[ES1] L. C. Evans and J. Spruck, Motion of level sets by mean curvature, I, J. Differential Geom. 33 (1991), 635-681.

[ES2] _ Motion of level sets by mean curvature. II, Trans. Amer. Math. Soc. 330 (1992), 321-332.

[GGIS] Y. Giga, S. Goto, H. Ishii, and M. H. Sato, Comparison principle and convexity preserving properties for singular degenerate parabolic equations on unbounded domains, Indiana Univ. Math. J. 40 (1990), 443-469.

[IKO] A. Ilin, A. S. Kalashnikov, and O. A. Oleiknik, Second-order linear equations of parabolic type, Russian Math. Surveys 17 (1962), 1-143.

[IL] H. Ishii and P. L. Lions, Viscosity solutions of fully nonlinear second-order elliptic partial differential equations, J. Differential Equations 83 (1990), 26-78.

[LSU] O. A. Ladyzhenskaya, V. A. Solonnikov, and N. N. Ural'ceva, Linear and quasilinear equations of parabolic type, Transl. Math. Monographs, Amer. Math. Soc., Providence, R.I., 1968.

[L] P. L. Lions, Generalized solutions of Hamilton-Jacobi equations, Res. Notes Math., vol. 69, Pitman, 1982.

[LSV] P. L. Lions, P. E. Souganidis, and J. L. Vázquez, The relation between the porous medium and the eikonal equations in several space dimensions, Rev. Mat. Iberoamericana 3 (1987), 275-310.

[M] P. Marcati, Approximate solutions to conservation laws via convective parabolic equations, Comm. Partial Differential Equations 13 (1988), 321-344.

[Mu] F. Murat, L'injection du cône positif de $H^{-1}$ dans $W^{-1, q}$ est compacte pour tout $q<2$, J. Math. Pures Appl. 60 (1981), 309-322.

[RM] R. D. Richtmyer and K. W. Morton, Difference methods for initial value problems, Interscience, New York, 1967.

[S] J. Serrin, Gradient estimates for solutions of nonlinear elliptic and parabolic equations, Contribution to Nonlinear Functional Analysis (E. H. Zarantonello, ed.), Academic Press, New York, 1971, pp. 565-601. SPAIN

Departamento de Matemáticas, Universidad Autónoma de Madrid, 28049 Madrid,

E-mail address: esteban@ccuam3.sdi.uam.es

Dipartimento di Matematica Pura ed Applicata, Universita dell'Aquila, 67100 L'AQUILA, ITALY

E-mail address: marcati@smaq20.univaq.it 